\title{
Performance of adenosine "stress-only" perfusion MRI in patients without a history of myocardial infarction: a clinical outcome study
}

\author{
Daniel D. Lubbers • Dorine Rijlaarsdam-Hermsen • \\ Dirkjan Kuijpers • Marjan Kerkhof • Paul E. Sijens • \\ Paul R. M. van Dijkman • Matthijs Oudkerk
}

Received: 14 July 2010/Accepted: 15 December 2010/Published online: 30 January 2011

(C) The Author(s) 2011. This article is published with open access at Springerlink.com

\begin{abstract}
To assess the diagnostic value of adenosine "stress-only" myocardial perfusion MR for ischemia detection as an indicator for coronary angiography in patients without a prior myocardial infarction and a necessity to exclude ischemia. Adenosine perfusion MRI was performed at $1.5 \mathrm{~T}$ in 139 patients with a suspicion of ischemia and no prior myocardial infarction. After 3 min of adenosine infusion a perfusion sequence was started. Patients with a perfusion defect were referred to coronary angiography (CAG). Patients with a normal perfusion were enrolled in follow-up.
\end{abstract}

D. D. Lubbers and D. Rijlaarsdam-Hermsen contributed equally to the manuscript.

D. D. Lubbers $(\bowtie) \cdot$ D. Kuijpers · P. E. Sijens ·

M. Oudkerk

Department of Radiology, University Medical Center

Groningen, University of Groningen, Groningen,

The Netherlands

e-mail: d.d.lubbers@rad.umcg.nl

D. Rijlaarsdam-Hermsen · P. R. M. van Dijkman

Department of Cardiology, Bronovo Hospital, The Hague,

The Netherlands

D. Kuijpers

Department of Radiology, Bronovo Hospital, The Hague, The Netherlands

\section{Kerkhof}

The Department of Epidemiology, University Medical Center Groningen, University of Groningen, Groningen, The Netherlands
Fourteen out of 139 patients $(10.1 \%)$ had a perfusion defect indicative of ischemia. These patients underwent a coronary angiogram, which showed complete agreement with the perfusion images. 125 patients with a normal myocardial perfusion entered follow-up (median 672 days, range 333-1287 days). In the first year of follow-up one Major Adverse Coronary Event (MACE) occurred and one patient had new onset chest pain with a confirmed coronary stenosis. Reaching a negative predictive value for MACE of $99.2 \%$ and for any coronary event of $98.4 \%$. At 2 year follow-up no additional MACE occurred. Sensitivity of adenosine perfusion MR for MACE is $93.3 \%$ and specificity and positive predictive value are $100 \%$. Adenosine myocardial perfusion MR for the detection of myocardial ischemia in a "stress-only" protocol in patients without prior myocardial infarctions, has a high diagnostic accuracy. This fast examination can play an important role in the evaluation of patients without prior myocardial infarctions and a necessity to exclude ischemia.

Keywords Cardiac $\cdot$ MRI $\cdot$ Ischemia $\cdot$ Perfusion

\section{Introduction}

Adenosine "stress" MR myocardial perfusion imaging has a proven high sensitivity and negative predictive value for the detection of myocardial ischemia [1-12]. High diagnostic accuracies are reached in patient groups with relatively high 
prevalence of disease in studies combining rest-stress perfusion and delayed contrast enhancement. For the subgroup of patients with a history of myocardial infarction these elaborate protocols or different stress MR imaging methods are probably most appropriate. In relatively lower risk patients, those without known myocardial infarction, less comprehensive protocols may be sufficient to guide further work-up and therapy choice. In lower-risk patient groups examined by adenosine "stress-only" perfusion MR imaging the number of purely diagnostic Coronary angiographies (CAG's) might thus be reduced, which would be important because CAG is an invasive test with a risk of complications and relatively expensive. Furthermore, taking into account that PCI with stent implantation is not harmless, invasive treatment should only be reserved for those patients with objectified myocardial ischemia [13, 14]. A noninvasive imaging technique such as an appropriately designed MR protocol, can be used as an indicator to determine which patients need to be directed to coronary angiography. With the routine implementation of adenosine perfusion MR still lagging behind, we sought to tailor a protocol designed for a specific population.

Directing the patient to the proper MR stress perfusion test or protocol, could yield diagnostic gain and time savings allowing analysis of larger patient groups.

In this study, the prognosis after a negative adenosine perfusion MR examination and the diagnostic accuracy of adenosine "stress" myocardial perfusion MR were examined in a stress-only approach, in patients without prior myocardial infarction and a clinical necessity to exclude myocardial ischemia.

\section{Methods}

Patient population

150 consecutive patients referred between January 2005 to April 2006 from the outpatient clinic of the department of Cardiology for an adenosine perfusion MR, were included. Eleven patients were not enrolled in the final study population due to a history of myocardial infarction ( 3 patients), use of vasoactive medication during adenosine ( 2 patients), moving out of the country with loss of follow-up ( 3 patients) and refused consent ( 3 patients). The final study population therefore consisted of 139 prospectively enrolled patients. The study was approved by the medical ethical board. Pre-test likelihood of these patients was determined according to a classification for chronic stable chest pain by Gibbons et al. [15]. Patients who could not be determined according to this classification were stratified with a calcium score (22 patients, mean calcium score $220( \pm 322)$ ) or considered to be at intermediate risk (for example rhythm abnormalities). Patients with a calciumscore $>90$ th percentile were considered to be at intermediate risk. Furthermore the percentage of patients with: hypertension, diabetes, smoking history, positive family history for coronary artery disease (CAD) and hypercholesterolemia and summary values on age, gender distribution, body weight and Body Mass Index (BMI) are displayed in Table 1.

Patients with a perfusion defect were referred for CAG. Patients with a normal adenosine perfusion examination had clinical follow-up for at least 1 year.

\section{Adenosine perfusion MR}

All anti-anginal medication was stopped 4 days before the adenosine perfusion MR examination. Xhantine containing products like coffee, tea, chocolate, cola had to be stopped $24 \mathrm{~h}$ prior to the examination. Dypiridamol had to be stopped or was considered a contra-indication. Scanning was performed at $1.5 \mathrm{~T}$ using a magnetom Avanto MRI system (Siemens Medical Solutions, Erlangen, Germany). After the patient was positioned on the scanning table, intravenous access was established via an anticubital vein. Vector ECG monitoring leads, a 12 channel phased-array surface coil covering the heart, and a brachial blood pressure cuff were applied. A single lead ECG signal was continuously monitored on the MRI-console. Systolic and diastolic blood pressures and heart rate were recorded at baseline and during adenosine infusion.

After $3 \mathrm{~min}$ of adenosine infusion $(0.140 \mathrm{mg} / \mathrm{kg} /$ min) during the first pass of $0.1 \mathrm{mmol} / \mathrm{kg}$ gadopentetate dimeglumine with a flow rate of $5 \mathrm{ml} / \mathrm{s}$ flushed with $15 \mathrm{ml} 0.9 \% \mathrm{NaCL}$ (flow rate $5 \mathrm{ml} / \mathrm{s}$ ) a noselective saturation recovery perfusion sequence, with high SNR, CNR and inline display was started (typical parameters): TrueFisp: TR, 150.5/163.1 ms 
Table 1 Demographic and hemodynamic data
Values are expressed as mean $\pm \mathrm{SD}$, range or percentage

\begin{tabular}{ll}
\hline Variable & Mean or \% \\
\hline Age, years, mean & $60.7 \pm 10.5$ \\
Male (\%) & 54 \\
Body mass index, kg, mean & $26.3 \pm 3.9$ \\
Hypercholesterolaemia (\%) & 86.1 \\
Hypertension (\%) & 46.3 \\
Diabetes (\%) & 14.9 \\
Current smokers (\%) & 18.8 \\
Former smokers (\%) & 44.2 \\
Positive family history (\%) & 52.1 \\
Pre-test likelihood & \\
Very low (\%) & 2.2 \\
Low (\%) & 19.4 \\
Intermediate (\%) & 55.4 \\
High (\%) & 23.0 \\
Resting diastolic blood pressure, mmHg, mean & $83.1 \pm 10.2$ \\
Diastolic blood pressure under adenosine, mmHg, mean & $87.6 \pm 10.0$ \\
Resting systolic blood pressure, mmHg, mean & $153.3 \pm 25.2$ \\
Systolic blood pressure under adenosine, mmHg, mean & $146.4 \pm 23.1$ \\
Resting heart rate, bpm, mean & $76.6 \pm 16.0$ \\
Heart rate under adenosine, bpm, mean & $88.5 \pm 17.5$ \\
\hline
\end{tabular}

(single heart beat temporal resolution); TE $1.03 \mathrm{~ms}$; TI $100 / 103 \mathrm{~ms} ; \alpha 45 / 50^{\circ}$; FOV $300 \times 300$; slicethickness $6 \mathrm{~mm}$; matrix $76 \times 128$; iPAT 2. Acquisition of three short-axis slices. Typical inplane resolution $2.5 \times 2.5 \mathrm{~mm}$. During the examination a radiologist and a cardiologist were present in the MR suite, to monitor the condition of the patient and to evaluate the images immediately. Total duration of the protocol was approximately $15 \mathrm{~min}$.

Image analysis

Perfusion series were visually analysed by an experienced radiologist and cardiologist in consensus, using a 16 segment model [16]. A perfusion abnormality in at least two segments at consecutive planes of the left ventricle or one segment of the most apical slice, was used as an indication for CAG. Patients with a perfusion defect were examined by CAG within 3 weeks.

Analysis of the coronary angiograms was performed by an experienced cardiologist, blinded to the MR results. A significant coronary lesion was defined as a narrowing of $>50 \%$. The decision for a PCI or $\mathrm{CABG}$ was made in regular consultation with cardiac surgeons and interventional cardiologists.

\section{Follow-up}

Follow-up was completed in October 2008. The status of the patient was determined by review of the hospital records, contacting the patient's general physician or by a questionnaire after informed consent. Reported clinical events were confirmed by contact with the treating hospital. The date of the hospital visit, last visit to the general physician or the date of returning the questionnaire was used to calculate follow-up time.

Patients were observed for occurrence of MACE (Major Adverse Cardiac Events) and MACE including coronary artery revascularization after objectified ischemia as composite end point and classified as composite MACE. Occurrence of noncardiac mortality was documented: such cases were censored for MACE evaluation at the time of death. 


\section{Primary outcome}

The aim of this study was to assess the diagnostic accuracy of an adenosine "stress-only" perfusion MR examination in patients without a prior myocardial infarction as a clinical indicator for coronary angiography and to determine the prognosis after a normal adenosine perfusion MR examination. Confirmation of the adenosine perfusion MR results was done by detection of a significant coronary stenosis on CAG or with at least 1 year follow-up in case of a normal adenosine perfusion MR examination.

\section{Statistics}

Sensitivity, specificity, negative and positive predictive values were calculated, with confidence intervals. Baseline characteristics are given as mean or median with standard deviation or range for continuous variables and as number (\%) for categorical variables.

\section{Results}

139 consecutive patients entered the study. Mean age $60.7 \pm 10.5$, 54\% male. Demographic and hemodynamic data are listed in Table 1. During adenosine perfusion MR no major adverse reactions were seen in this patient group.

Fourteen out of 139 patients (10.1\%) had a perfusion abnormality indicative for myocardial ischemia, Fig. 1. Corresponding CAG displayed in Fig. 2.

On a per patient basis significant coronary artery disease was demonstrated by CAG in all fourteen patients, followed by revascularization in 10 patients (1 CABG, 9 PCI, in 4 patients revascularization was not feasible).

The 125 patients with a negative (normal) adenosine perfusion MR examination were followed up for a median period of 672 days (range 333-1,287 days). There was one MACE during the first follow-up year $(0.8 \%)$ due to an acute coronary syndrome complicated by ventricular fibrillation, 12 months after the adenosine perfusion MR examination. There was one case of new onset chest pain 10 months after the adenosine perfusion MR examination with subsequent

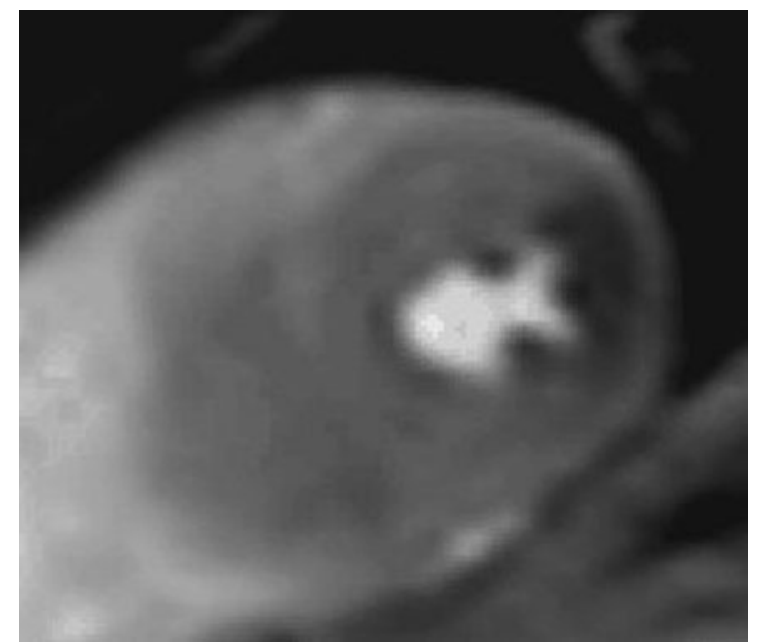

Fig. 1 Mid-ventricular short-axis single frame with perfusion defect in the distribution area of the LCX

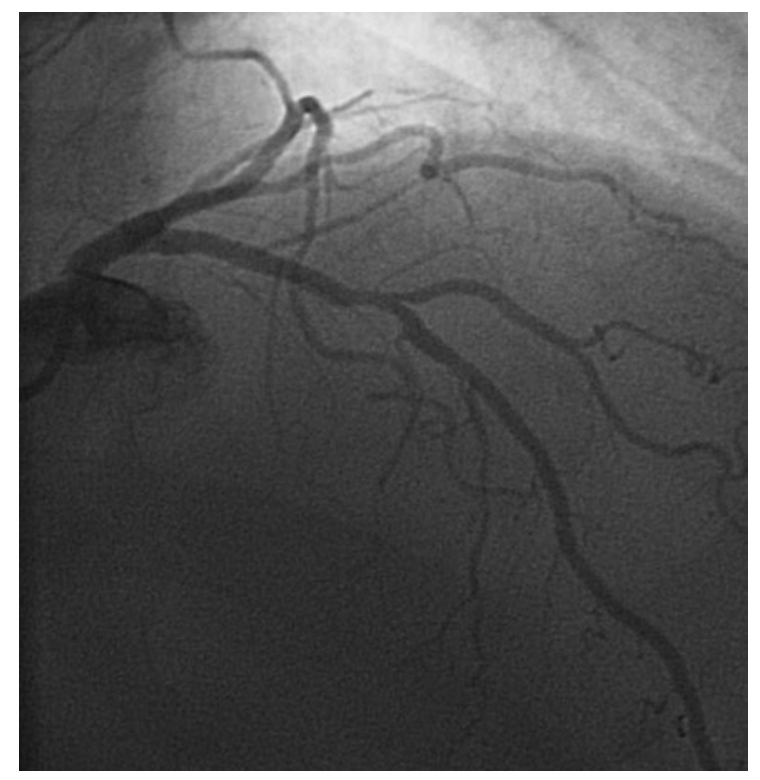

Fig. 2 Corresponding LCX stenosis on CAG

stent implantation, giving a composite MACE rate of $1.6 \%$. Both patients had a low-pre-test likelihood.

In the second follow-up year two additional revascularizations were performed (17 and 18 months after the adenosine perfusion MR and no additional MACE.

Diagnostic values for sensitivity are $93.3 \%$ (CI: 0.68-0.99), specificity $100 \%$ (CI: 0.97-1.00), 
Negative Predictive Value (NPV) 99.2 (CI: 0.961.00), and Positive Predictive Value (PPV) of $100 \%$ (CI: $0.77-1.00)$. Only 6 out of 32 patients (18.8\%) with a high pre-test likelihood had a positive adenosine perfusion MR examination.

Distribution of the pre-test likelihood of significant coronary artery disease is presented in Table 1.

\section{Discussion}

Main results of this study are that prognosis after a negative adenosine perfusion MR examination is good in this patient group and justifies conservative treatment rather than performing an invasive examination. Second, comparison between positive adenosine perfusion MR examinations and CAG is good on a per patient basis, and could be used in the preselection of patients to be examined by coronary angiography.

Diagnostic performances reported of adenosine perfusion MR studies, vary widely depending on the pulse-sequence, contrast dose, the modality used as a reference standard, the studied patient population and the used protocol. Besides this, coronary artery disease is a progressive disease, which to some extent explains the relative late occurrence of MACE or composite MACE after a negative adenosine perfusion MR in the few patients in this study. Results should in this regard also be seen as ongoing disease and not by definition as a false negative examination.

For a visual, qualitative approach to adenosine perfusion MR, one needs an imaging protocol approach. A good, but extensive approach has been proposed by Klem et al. [7]. Starting analysis with delayed contrast imaging, followed by rest and stress perfusion images. For a specific population without prior myocardial infarction we propose an imaging strategy that focuses on the adenosine stress perfusion MR series, to answer the question if there is a need for coronary angiography. In a relatively lower prevalence population the adenosine perfusion MR examination can exclude myocardial ischemia in a large group of patients with a normal adenosine "stress-only" perfusion examination, saving considerable imaging time and thus allowing analysis of larger patient groups. The importance of this study is underlined by a recent assessment by Nandalur et al.
[17] that relatively little knowledge is available on the use of stress perfusion imaging in lower pre-test probability groups such as in patients without prior myocardial infarction.

$\mathrm{CAG}$, an invasive, expensive test with a risk of complications, can in this strategy be reserved for patients with objectified ischemia. In this way adenosine perfusion MR can be used to reduce the number of pure diagnostic CAG's.

The few long-term follow-up studies published so far [1, 12, 18], found good prognosis for a negative adenosine perfusion MR examinations, results we can confirm with our study in this patient group. To the best of our knowledge this is the first study to assess the long term follow-up of an adenosine "stressonly" approach.

Different imaging modalities can serve as a gatekeeper for further invasive examinations. Exercise ECG testing results are less accurate than believed. A meta-analysis of 147 published reports with in total 24,074 patients reports a mean sensitivity of exercise ecg-testing of $68 \%$ and a mean specificity of $77 \%$ [15]. Diagnostic accuracy is even lower when the test is performed only in patients without a previous myocardial infarction.

Nuclear imaging modalities play an important role in many centers and a lot of experience and validation is present, but they do have some important drawbacks regarding limited spatial and temporal resolution, attenuation artefacts and the use of radiation. All issues that can be overcome with adenosine perfusion MR, but for MR to be able to compete with nuclear and other stress imaging modalities, imaging time needs to be short, images easily interpretable and protocols optimised for the patient population, taking into account the presence of a prior myocardial infarction, and the need for assessment of viable myocardium. On indication a rest perfusion MR examination or delayed contrast enhancement can be performed. The optimal population for an adenosine perfusion MR examination is in our opinion found in the patient group without a prior myocardial infarction. For patients with a prior myocardial infarction viability imaging may also be required.

Diagnostic performance of dobutamine stress MR examinations in this respect has shown good results, with a good long term prognosis [18-20]. Assessment of viability can be performed in the same 
examination in a functional way, without increasing imaging time significantly and may be more reliable than quantification of scar tissue [21]. In a patient group without prior myocardial infarction absence of myocardial ischemia can be determined with a normal, homogeneous, adenosine perfusion MR serie, with an imaging time of only $15 \mathrm{~min}$.

Some earlier studies have reported moderately high specificities, due to the fact that perfusion MR was not able to discriminate between perfusion defects caused by ischemia or other causes $[2,10]$. Specificity is probably also high in this study probably due to examining patients without a prior history of myocardial infarction and the use of clear set reading criteria [16].

The use of CAG as a reference standard might be a limitation, because CAG may be a "flawed"gold standard. CAG fails to account for the effect of diffuse disease, length of diseased segments and serial stenoses, and the functional effects in terms of perfusion for the myocardium [22]. Higher levels of diagnostic accuracy are observed when adenosine perfusion MR was compared with PET [23] or FFR measurements as the reference standard [24, 25]. Current clinical practice regarding risk stratification and therapy guidance is however directed by the CAG, which makes it a clinically relevant reference standard.

Rather than performing a quantitative analysis, we optimized the imaging protocol for a robust, visual approach. This can be regarded as a limitation, but previous studies have shown that quantitative and qualitative, visual assessment of myocardial perfusion to have similar good correlations with CAG [23, $26,27]$. Delayed contrast enhancement imaging or rest perfusion imaging was not routinely performed. This may provide additional valuable diagnostic information, but mostly in a post-infarct setting. This might therefore be regarded as a limitation, but was a choice made for a broad application of adenosine perfusion MR in a specific population in which we doubt that it is of additional value. Prior myocardial infarction, as stated earlier, was used as an exclusion criterion in this study.

\section{Conclusions}

Adenosine perfusion MR, in a "stress"-only approach has a high diagnostic accuracy and may have a distinct clinical role in patients without previous myocardial infarctions as an examination which can reliably determine the necessity for coronary angiography in a total protocol time of only $15 \mathrm{~min}$.

Acknowledgments The authors thank Bernadette Blom and colleagues for image acquisition and Stella Noach for critically reviewing the paper and Dr. J. Frederiks for analysing the CAG's.

Conflict of interest None declared.

Open Access This article is distributed under the terms of the Creative Commons Attribution Noncommercial License which permits any noncommercial use, distribution, and reproduction in any medium, provided the original author(s) and source are credited.

\section{References}

1. Ingkanisorn WP, Kwong RY, Bohme NS, Geller NL, Rhoads KL, Dyke CK, Paterson DI, Syed MA, Aletras AH, Arai AE (2006) Prognosis of negative adenosine stress magnetic resonance in patients presenting to an emergency department with chest pain. J Am Coll Cardiol 47(7): 1427-1432

2. Sakuma H, Suzawa N, Ichikawa Y, Makino K, Hirano T, Kitagawa K, Takeda K (2005) Diagnostic accuracy of stress first-pass contrast-enhanced myocardial perfusion MRI compared with stress myocardial perfusion scintigraphy. AJR Am J Roentgenol 185(1):95-102

3. Plein S, Radjenovic A, Ridgway JP, Barmby D, Greenwood JP, Ball SG, Sivananthan MU (2005) Coronary artery disease: myocardial perfusion MR imaging with sensitivity encoding versus conventional angiography. Radiology 235(2):423-430

4. Giang TH, Nanz D, Coulden R, Friedrich M, Graves M, Al-Saadi N, Luscher TF, von Schulthess GK, Schwitter J (2004) Detection of coronary artery disease by magnetic resonance myocardial perfusion imaging with various contrast medium doses: first European multi-centre experience. Eur Heart J 25(18):1657-1665

5. Paetsch I, Foll D, Langreck H, Herkommer B, Klein C, Schalla S, Fleck E, Nagel E (2004) Myocardial perfusion imaging using OMNISCAN: a dose finding study for visual assessment of stress-induced regional perfusion abnormalities. J Cardiovasc Magn Reson 6(4):803-809

6. Bernhardt P, Engels T, Levenson B, Haase K, Albrecht A, Strohm O (2006) Prediction of necessity for coronary artery revascularization by adenosine contrast-enhanced magnetic resonance imaging. Int J Cardiol 112(2):184-190

7. Klem I, Heitner JF, Shah DJ, Sketch MH Jr, Behar V, Weinsaft J, Cawley P, Parker M, Elliott M, Judd RM, Kim RJ (2006) Improved detection of coronary artery disease by stress perfusion cardiovascular magnetic 
resonance with the use of delayed enhancement infarction imaging. J Am Coll Cardiol 47(8):1630-1638

8. Pilz G, Bernhardt P, Klos M, Ali E, Wild M, Hofling B (2006) Clinical implication of adenosine-stress cardiac magnetic resonance imaging as potential gatekeeper prior to invasive examination in patients with AHA/ACC class II indication for coronary angiography. Clin Res Cardiol 95(10):531-538

9. Cury RC, Cattani CA, Gabure LA, Racy DJ, de Gois JM, Siebert U, Lima SS, Brady TJ (2006) Diagnostic performance of stress perfusion and delayed-enhancement MR imaging in patients with coronary artery disease. Radiology 240(1):39-45

10. Wolff SD, Schwitter J, Coulden R, Friedrich MG, Bluemke DA, Biederman RW, Martin ET, Lansky AJ, Kashanian F, Foo TK, Licato PE, Comeau CR (2004) Myocardial firstpass perfusion magnetic resonance imaging: a multicenter dose-ranging study. Circulation 110(6):732-737

11. Plein S, Greenwood JP, Ridgway JP, Cranny G, Ball SG, Sivananthan MU (2004) Assessment of non-ST-segment elevation acute coronary syndromes with cardiac magnetic resonance imaging. J Am Coll Cardiol 44(11):2173-2181

12. Pilz G, Jeske A, Klos M, Ali E, Hoefling B, Scheck R, Bernhardt P (2008) Prognostic value of normal adenosinestress cardiac magnetic resonance imaging. Am J Cardiol 101(10):1408-1412

13. Bech GJ, De Bruyne B, Pijls NH, de Muinck ED, Hoorntje JC, Escaned J, Stella PR, Boersma E, Bartunek J, Koolen JJ, Wijns W (2001) Fractional flow reserve to determine the appropriateness of angioplasty in moderate coronary stenosis: a randomized trial. Circulation 103(24):2928-2934

14. Kern MJ, Lerman A, Bech JW, De Bruyne B, Eeckhout E, Fearon WF, Higano ST, Lim MJ, Meuwissen M, Piek JJ, Pijls NH, Siebes M, Spaan JA (2006) Physiological assessment of coronary artery disease in the cardiac catheterization laboratory: a scientific statement from the American heart association committee on diagnostic and interventional cardiac catheterization, council on clinical cardiology. Circulation 114(12):1321-1341

15. Gibbons RJ, Balady GJ, Beasley JW, Bricker JT, Duvernoy WF, Froelicher VF, Mark DB, Marwick TH, McCallister BD, Thompson PD, Winters WL Jr, Yanowitz FG, Ritchie JL, Cheitlin MD, Eagle KA, Gardner TJ, Garson A Jr, Lewis RP, O'Rourke RA, Ryan TJ (1997) ACC/AHA guidelines for exercise testing: executive summary. A report of the American college of cardiology/american heart association task force on practice guidelines (committee on exercise testing). Circulation 96(1):345-354

16. Lubbers DD, Kuijpers D, Bodewes R, Kappert P, Kerkhof M, van Ooijen PM, Oudkerk M (2010) Inter-observer variability of visual analysis of "stress"-only adenosine first-pass myocardial perfusion imaging in relation to clinical experience and reading criteria. Int $\mathrm{J}$ Cardiovasc Imaging EPUB ahead of print

17. Nandalur KR, Dwamena BA, Choudhri AF, Nandalur MR, Carlos RC (2007) Diagnostic performance of stress cardiac magnetic resonance imaging in the detection of coronary artery disease: a meta-analysis. J Am Coll Cardiol 50(14): 1343-1353

18. Jahnke C, Nagel E, Gebker R, Kokocinski T, Kelle S, Manka R, Fleck E, Paetsch I (2007) Prognostic value of cardiac magnetic resonance stress tests: adenosine stress perfusion and dobutamine stress wall motion imaging. Circulation 115(13):1769-1776

19. Kuijpers D, van Dijkman PR, Janssen CH, Vliegenthart R, Zijlstra F, Oudkerk M (2004) Dobutamine stress MRI. Part II. Risk stratification with dobutamine cardiovascular magnetic resonance in patients suspected of myocardial ischemia. Eur Radiol 4(11):2046-2052

20. Hundley WG, Hamilton CA, Thomas MS, Herrington DM, Salido TB, Kitzman DW, Little WC, Link KM (1999) Utility of fast cine magnetic resonance imaging and display for the detection of myocardial ischemia in patients not well suited for second harmonic stress echocardiography. Circulation 100(16):1697-1702

21. Wellnhofer E, Olariu A, Klein C, Grafe M, Wahl A, Fleck E, Nagel E (2004) Magnetic resonance low-dose dobutamine test is superior to SCAR quantification for the prediction of functional recovery. Circulation 109(18): 2172-2174

22. Crean A, Merchant N (2006) MR perfusion and delayed enhancement imaging in the heart. Clin Radiol 61(3): 225-236

23. Schwitter J, Nanz D, Kneifel S, Bertschinger K, Buchi M, Knusel PR, Marincek B, Luscher TF, von Schulthess GK (2001) Assessment of myocardial perfusion in coronary artery disease by magnetic resonance: a comparison with positron emission tomography and coronary angiography. Circulation 103(18):2230-2235

24. Rieber J, Huber A, Erhard I, Mueller S, Schweyer M, Koenig A, Schiele TM, Theisen K, Siebert U, Schoenberg SO, Reiser M, Klauss V (2006) Cardiac magnetic resonance perfusion imaging for the functional assessment of coronary artery disease: a comparison with coronary angiography and fractional flow reserve. Eur Heart J 27(12):1465-1471

25. Watkins S, McGeoch R, Lyne J, Steedman T, Good R, McLaughlin MJ, Cunningham T, Bezlyak V, Ford I, Dargie HJ, Oldroyd KG (2009) Validation of magnetic resonance myocardial perfusion imaging with fractional flow reserve for the detection of significant coronary heart disease. Circulation 120(22):2207-2213

26. Ishida N, Sakuma H, Motoyasu M, Okinaka T, Isaka N, Nakano T, Takeda K (2003) Noninfarcted myocardium: correlation between dynamic first-pass contrast-enhanced myocardial MR imaging and quantitative coronary angiography. Radiology 229(1):209-216

27. Chiu CW, So NM, Lam WW, Chan KY, Sanderson JE (2003) Combined first-pass perfusion and viability study at MR imaging in patients with non-ST segment-elevation acute coronary syndromes: feasibility study. Radiology 226(3):717-722 